\title{
A Novel Self-Powered Approach for Structural Health Monitoring
}

\author{
Amir H. Alavi, Hassene Hasni, Nizar Lajnef \\ Department of Civil and Environmental Engineering, \\ Michigan State University, East Lansing, \\ MI 48823, USA \\ Email: lajnefni@msu.edu
}

\author{
Sami Masri \\ Sonny Astani Department of Civil and Environmental \\ Engineering, University of Southern California, Los Angeles, \\ CA 90089, USA \\ Email: masri@usc.edu
}

\begin{abstract}
- this computational simulation study presents an innovative approach for structural damage detection in "smart" civil infrastructure systems. The proposed approach is predicated upon the utilization of the compressed data stored in memory chips of a newly developed self-powered wireless sensor. An efficient data interpretation system, integrating aspects of the finite element method (FEM) and probabilistic neural networks (PNN) based on Bayesian decision theory, is developed for damage detection. Several features extracted from the cumulative limited static strain data are used as damage indicator variables. The efficiency of the method is tested and evaluated for the complicated case of a bridge gusset plate. The gusset plate structure is analysed via 3D FE models. A general scheme is presented for finding the optimal number of data acquisition points (sensors) on the structure and the associated optimal locations, taking into account the influence of sensor sparsity and the level of data corruption due to noise.
\end{abstract}

Keywords-t structural health monitoring, selfpowered wireless sensor, damage detection, uncertainty analysis, probabilistic neural networks, data summarization.

\section{INTRODUCTION}

In the last decade, significant attention has been devoted to the utilization of new sensing technologies for structural health monitoring (SHM). A major drawback of using traditional wired sensors pertains to the difficulties in deploying and maintaining the associated wiring system. To cope with these limitations, wireless sensor networks (WSNs) are increasingly utilized as alternatives to traditional structural engineering monitoring systems. Dense arrays of low-cost smart wireless sensors can offer useful data about the associated structural deterioration. However, a major concern for the application of wireless sensors is about their power supply. Harvesting ambient energy seems to be an attractive solution for tackling this issue [1]. Energy harvesting relies on the possibility of converting mechanical energy into electrical energy [2]. Recently, the authors have developed a new class of selfpowered wireless sensors (SWS) [3]. The developed SWS is a small size battery-less sensor. The prototype of this miniaturized strain-senor is shown in Fig. 1. This unique sensor is based on the integration of the piezoelectric (PZT) transducers with an array of ultra-low power floating gate computational circuits [4]. This sensor has a series of memory cells that store the load history profiles transferred to an accompanying data logger system as a voltage signal through the piezoelectric effect. Each of the memory cells records events at a specific preset level. The output is reported in the form of a histogram where each bin represents the cumulative time of occurrences at a predetermined load level.

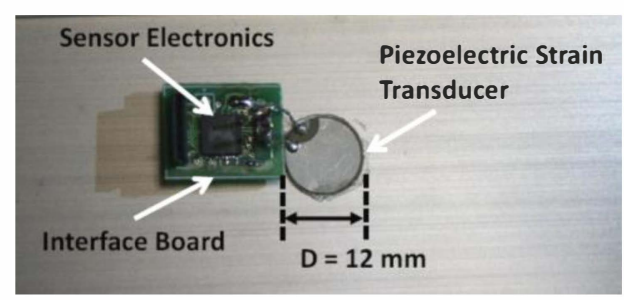

Fig. 1. The prototype of the SWS system.

This study presents a new methodology for structural damage detection based on the simulation of the SWS data. The proposed approach uses features extracted from the cumulative time strain distributions at preselected discrete levels. In order to analyze the cumulative static strain data, a hybrid method involving probabilistic neural networks (PNN) and FE is proposed. PNN is a neural network implementation of the well-established Bayesian classifier method [5]. The FE model of a gusset plate with complex (and realistic) geometry components is used as a representative model of real structures for the proposed method verification. 


\section{THE Proposed METHOdOLOGY}

As discussed above, the new smart SWS made at MSU is capable of continuously monitoring local strains within the host structure. These sensors have a series of memory cells that cumulatively store the duration of strain events, at a preselected level of discretization. They measure the duration of events when the amplitude of the input signal, coming from the piezo, exceeds different thresholds. At a constant loading frequency, the sensor output is the cumulative histogram of the loading strain distribution. Figure 2 presents a schematic representation of the level crossing cumulative time counting implemented by the developed SWS.

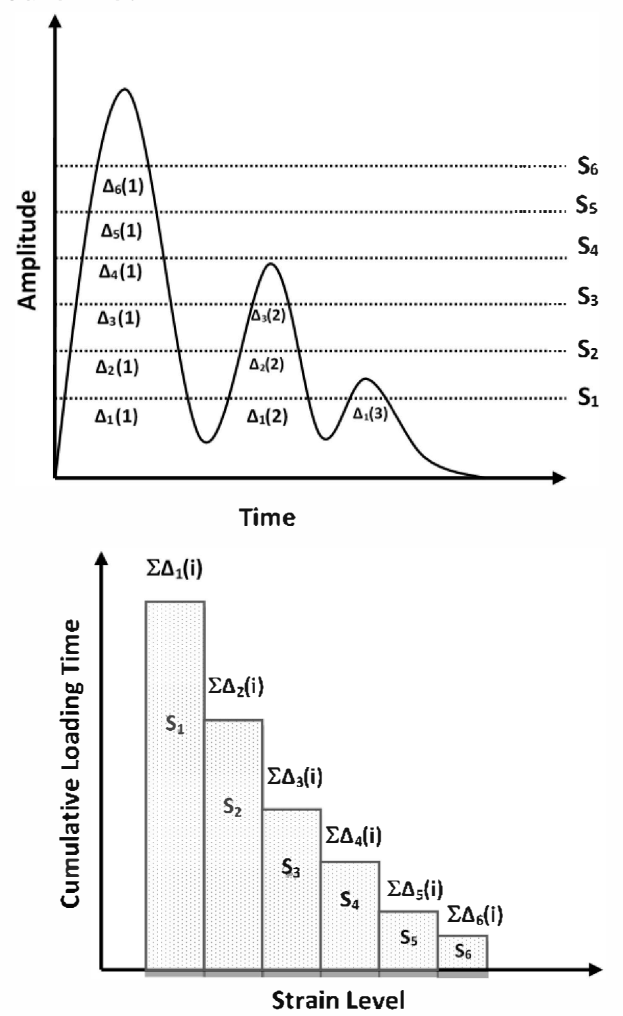

Fig. 2. The level-crossing cumulative time counting implemented by the SWS

With a Gaussian distributed load, the sensor output is the summation of the cumulative time strain distributions. Since the summation of Gaussian distributions has itself a Gaussian distribution, this study rationally assumes that the sensor output can be characterized by the following cumulative density function (CDF):

$$
F(\varepsilon)=\frac{\alpha}{2}\left[1-\operatorname{erf}\left(\frac{\varepsilon-\mu}{\sigma \sqrt{2}}\right)\right]
$$

where

$\mu$ : Mean of the strain distribution

$\sigma$ : Standard deviation that accounts for the load and frequency variability

$\alpha$ : Total cumulative time of the measured strain.
On the basis of previous research [4], $\mu$ and $\sigma$ of the strain distribution can be regarded as good indicators of the damage progression. In fact, $\mu$ and $\sigma$ are the only viable tools to define the SWS output data. These parameters can be obtained by a curve fitting of the sensor output distribution collected from the entire memory cells. Consequently, the damage state is logically considered to be a function of $\mu$ and $\sigma$. In the present study, the cumulative time of occurrences at predetermined strain levels are determined for each of the sensors. Then, the corresponding $\mu$ and $\sigma$ values for each damage scenario were obtained through the fitting of a CDF (Eq. (1)) to the cumulative duration of strain events.

\section{CASE EXAMPLE - PERFORMANCE VERIFICATION}

A series of FE simulations are conducted to extract the static strain measurements at specified or randomly selected locations. Herein, the structures under consideration are assumed to be linearly elastic and are analyzed as 3D FE models using Abaqus. The input loading type is in the form of half-sine loading. The 3D FE model for the gusset plate is shown in Fig. 3. The load magnitude is taken equal to $10 \%$ of the critical loading at the time of bridge collapse [6]. The plate is modeled using three dimensional linear tetrahedral elements (C3D4). The model of the gusset plate consists of about 50000 C3D4 elements corresponding to about 25000 nodes. However, the material and geometry properties used in the model are: $\mathrm{E}=200000 \mathrm{MPa}$ (Steel ASTM-A36); Poisson Ratio $=0.3$; Density $=7800 \mathrm{~kg} / \mathrm{m}^{3}$; Load Frequency $=0.5 \mathrm{~Hz}$. The thickness of the gusset plate is 0.5 inch $(12.7 \mathrm{~mm})$. The diameter for the data acquisition nodes (potential sensors) is equal to $10 \mathrm{~mm}$. The average of the maximum principle strains at the nodes, within the sensor specified area, is taken as the representative strain value for each sensor. Several sensors are considered for the analysis. The distance between the sensors is taken as $80 \mathrm{~mm}$. Considering the dimension of the plate, 28 and 16 sensors are defined in the horizontal and vertical directions, respectively. Thus, the maximum number of data acquisitions nodes is $28 \times 16=448$.

Taking into account the final length of the crack at the plate failure $(530 \mathrm{~mm})$ and its direction, fifteen damage states (classes) are defined. The damage is simulated by creating notches of different sizes through a new series of $\mathrm{FE}$ analyses. The first damaged case belongs to the initial notch with $10 \mathrm{~mm}$ length. The other states are created by adding $20 \mathrm{~mm}$ to each side of the initial notch on the direction already detected by extended finite element method (XFEM). For instance, the third damaged class pertains to the notch with an initial length of $10 \mathrm{~mm}$ plus 
$20 \mathrm{~mm}$ on each side, $10+20+20=60 \mathrm{~mm}$. Subsequently, the damage classes can be defined by changing the notch size (a) as follows: 1: Intact beam $(a=0 \mathrm{~mm}) ; 2: a=10$ $\mathrm{mm}$ (Initial notch); 3: a $=50 \mathrm{~mm} ; 4: \mathrm{a}=90 \mathrm{~mm} ; 5: \mathrm{a}=$ $130 \mathrm{~mm}$; $6: \mathrm{a}=170 \mathrm{~mm} ; 7: \mathrm{a}=210 \mathrm{~mm} ; 8: \mathrm{a}=250 \mathrm{~mm}$; 9: $\mathrm{a}=290 \mathrm{~mm} ; 10: \mathrm{a}=330 \mathrm{~mm} ; 11: \mathrm{a}=370 \mathrm{~mm} ; 12: \mathrm{a}=$ $410 \mathrm{~mm} ; 13: \mathrm{a}=450 \mathrm{~mm} ; 14: \mathrm{a}=490 \mathrm{~mm} ; 15: \mathrm{a}=530$ $\mathrm{mm}$; The minimum level of strains needed to be captured by piezoelectric transducers is about $30.00 \mu \varepsilon$. On the other hand, the maximum strain value extracted from the FE simulations of fifteen damage scenarios is about 150 $\mu \varepsilon$. Considering a reasonable number of ten strain levels for the gates, the difference between the strain levels is $13.33 \mu \varepsilon$. For this complicated case, the analysis approach takes the strain-time data from Abaqus and finds the strain information for the considered 448 (or any other) number of sensors with different sizes (for this case: $10 \mathrm{~mm}$ ).

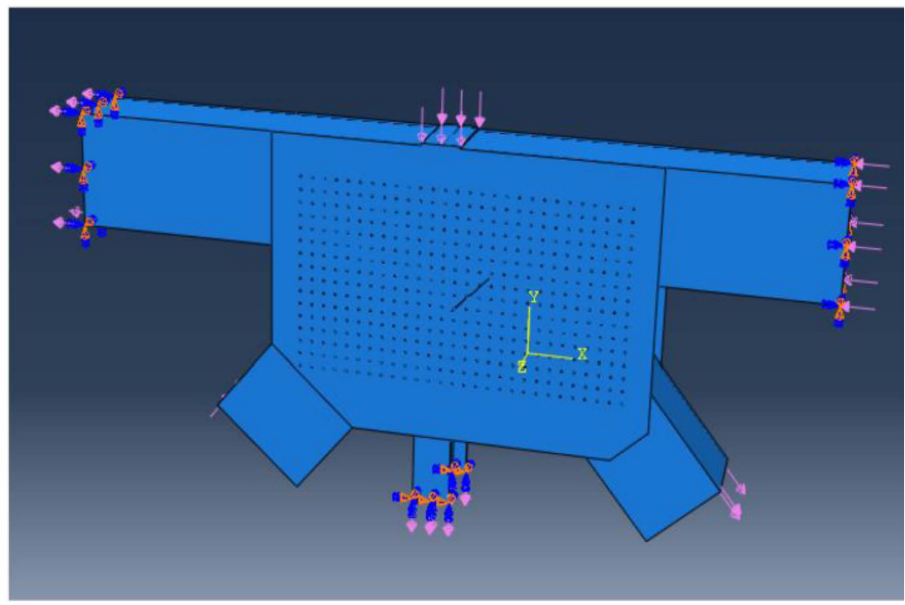

Fig. 3. The geometry of the gusset plate

The PNN algorithm is used for the damage state. The PNN algorithm adopts a Bayes decision rule.The PNN architecture consists of four layers: (1) input layer, (2) pattern layer, (3) summation layer, and (4) a single-node output layer. In addition to the randomized configuration of the sensors, the organized positioning of the sensors is also considered. A maximum of 224 sensors is taken into account. The analyses are performed with $224,112,56$, and 28 organized and randomly selected sensors. Different pre-determined configuration schemes with 24 , $28,30,42,53,57$, and 64 sensors are also considered to assess the importance of locating the sensors at probable critical regions. Figure 4 shows a schematic representation of the sensor configurations. In this figure, black and red cells represent the sensors. The red cells show the sensors that do not sense damage in all of the damage states. Since such sensors do not record any information, they are excluded from the analyses. The black cells define the active sensors.

It is decided to use both the information provided by one sensor and also the general information supplied by the other sensors in that specific sensor configuration (group of sensors). In this case, even if one sensor does not sense the damage, the group effect will help detect the damage. To tackle this issue, a set of new input parameters are introduced to the formulation of the damage state as follows:

\section{Damagestate $=$}

$f\left(X, Y, \mu, \sigma, \mu_{D}, \sigma_{D}, \mu_{S}, \sigma_{S}, Z_{\mu 1}, Z_{\sigma 1}, Z_{\mu 2}, Z_{\sigma 2}\right)$

where,

$\mu_{\mathrm{D}}: \frac{\mu}{\mu_{\mathrm{ave}}}$ and $\mu_{\mathrm{ave}}$ is the average of $\mu$ of all sensors for a specific damage scenario that $\mu$ belongs to.

$\sigma_{\mathrm{D}}: \frac{\sigma}{\sigma_{\text {ave }}}$ and $\sigma_{\text {ave }}$ is the average of $\sigma$ of all sensorsfor a specific damage scenario that $\sigma$ belongs to.

$\mu_{\mathrm{s}}: \mu-\mu_{\mathrm{ave}}$ and $\mu_{\mathrm{ave}}$ is the average of $\mu$ of all sensors for a specific damage scenario

$\sigma_{\mathrm{S}}: \sigma-\sigma_{\mathrm{ave}}$ and $\sigma_{\mathrm{ave}}$ is the average of $\sigma$ of all sensors for a specific damage scenario

$Z_{\mu 1}: \frac{\mu-\mu_{\mathrm{ave}}}{\mu_{\mathrm{STD}}}$ and $\mu_{\mathrm{STD}}$ is the standard deviation of $\mu$ of all sensors for a specific damage scenario

$Z_{\sigma 1}: \frac{\sigma-\sigma_{\mathrm{ave}}}{\sigma_{\mathrm{STD}}}$ and $\sigma_{\mathrm{STD}}$ is the standard deviation of $\sigma$ of all sensors for a specific damage scenario

$Z_{\mu 2}: \frac{\mu-\mu_{\text {ave }}}{\sigma_{\text {ave }}}$

$Z_{\sigma 2}: \frac{\sigma-\mu_{\text {ave }}}{\sigma_{\text {ave }}}$

Note that $Z_{\mu 1}$ and $Z_{\sigma 1}$ are z-score functions. $Z_{\mu 2}$ and $Z_{\sigma 2}$ are functions defined in this study inspired by the form of the conventional z-score function. As can be observed, the considered input variables efficiently take into account the sensor group effect that would improve the detection performance, specifically for low number of sensors. After extensive preliminary runs, it was found that $\mathrm{X}, \mathrm{Y}$, $\mu$, and $\sigma$ cannot be solely used for damage detection. Moreover, among different defined input features, $Z_{\mu 1}, Z_{\sigma 1}, Z_{\mu 2}$, and $Z_{\sigma 2}$ are the most efficient damage indicator variables. Consequently, these four input features are used for detecting the damage states introduced into the gusset plate. The available data for each of the sensor configurations are randomly divided into three calibration ( $70 \%$ of the data), validation $(15 \%$ of the data), and testing ( $15 \%$ of the data) subsets. For each of the randomized sensor configurations, a number 


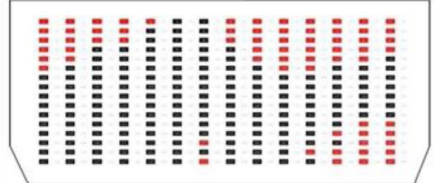

224 Organized Sensors (156 Active)

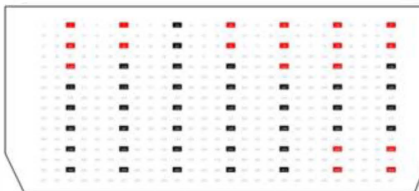

56 Organized Sensors (37 Active)

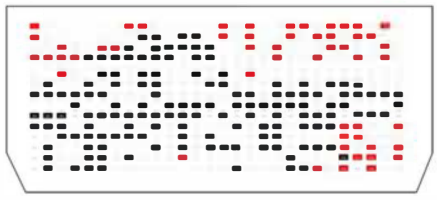

224 Randomized Sensors (168 Active)

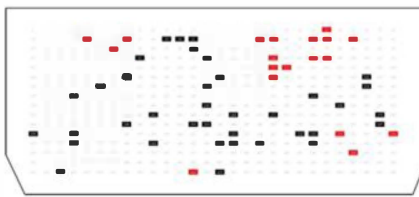

56 Randomized Sensors (37 Active)

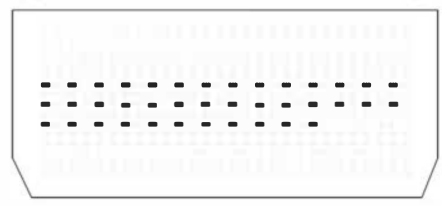

39 Sensors at Specified Locations

28 Sensors at Specified Locations

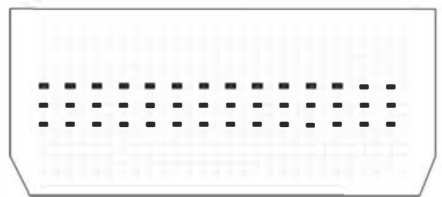

42 Sensors at Specified Locations

53 Sensors at Specified Locations

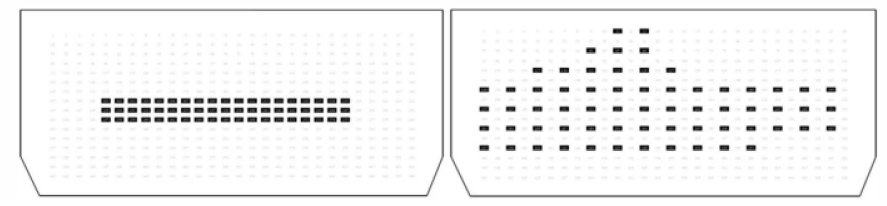

57 Sensors at Specified Locations

(n) 64 Sensors at Specified Locations

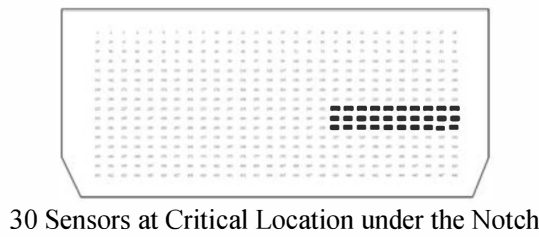

Fig. 4. A schematic representation of the sensor configurations for the gusset plate of repetitions are conducted to guarantee that the models with the best performance on both the calibration and validation phases are developed. Obviously, this issue is of great importance for a case involving a low number of sensors.

A Gaussian function is adopted as the window function for the PNN algorithm. The best classification results for different number of sensors are presented in Table 1. In order to have an insight into the area covered by the sensors, the ratio of the area of sensors in each configuration to the area of the plate (Area Ratio) is also shown in Table 1. As shown, the model with 156, 75, 37, and 17 organized sensors have a very good performance on the calibration, validation and testing data. The performance of the models built for 168 and 72 randomly selected sensors is also very satisfying. As expected, the precision of the models decreases with a decreasing number of sensors. However, considering the Area Ratio values, it is seen that even for 168 randomized sensors, only $0.39 \%$ of the plate is covered by the sensors (less than $1 \%$ ). This is indeed a very low rate for achieving such high detection accuracy. On the other hand, for the predetermined configuration schemes, only 64 and 30 sensors provide acceptable results. This indicates that organized and randomized distribution of the sensors can be a more effective strategy for increasing the detection precision. It is worth mentioning that, by decreasing the number of damage classes, the accuracy of the models remarkably increases. For instance, the detection accuracy of the 17 organized sensors for 8 damage classes is equal to $100 \%, 85 \%$ and $90 \%$ on the calibration, validation and testing data, respectively. For the 37 active organized sensors, these values are, respectively, equal to $100 \%, 91 \%$ and $86 \%$ on the calibration, validation and testing data. Thus, it is possible to reduce the number of sensors and yet have good detection accuracy by decreasing the number of damage classes.

In order to analyze the uncertainties in predictions, the calibration, validation and testing sets are polluted with random noise. The noise pollution verification phase has been done only for the best models shown in Table 1 . That is, the models for $156,75,37$, and 17 organized sensors, 168 and 72 randomly selected sensors, and 64 sensors at specified locations are chosen for the uncertainty analysis. Cases of $5 \%, 10 \%, 20 \%$, and $30 \%$ noise levels are considered. The PNN algorithm is run for all the chosen combinations. Figure 5 presents the best classification results for different number of sensors with various noise levels for the testing data. Comparing the results shown in Fig. 5, it is seen that increasing the noise level even up to $30 \%$ does not significantly influence the 
performance of the models built for 168 and 72 randomly selected sensors. Also, the performance of the models for 156 and 75 organized sensors is acceptable up to about $20 \%$ noise level. In all cases, it can be seen that the identification accuracies of the models gradually decrease with the increase of the noise level. Besides, the model for 64 sensors at specified locations has an acceptable accuracy only for $10 \%$ noise level.

Table 1. The damage detection performance for the gusset plate

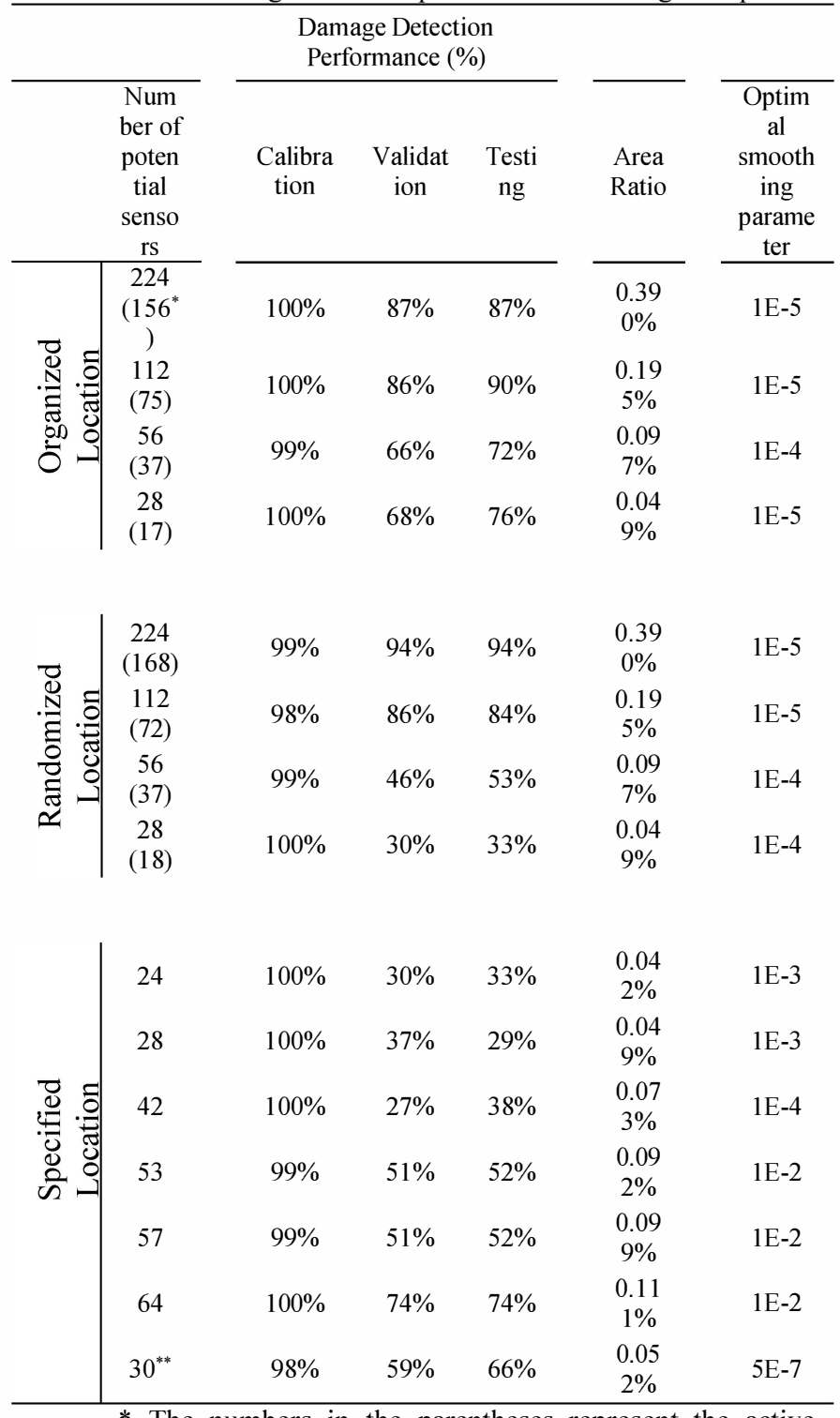

* The numbers in the parentheses represent the active sensors for each configuration.

**The sensors are located under the notch.

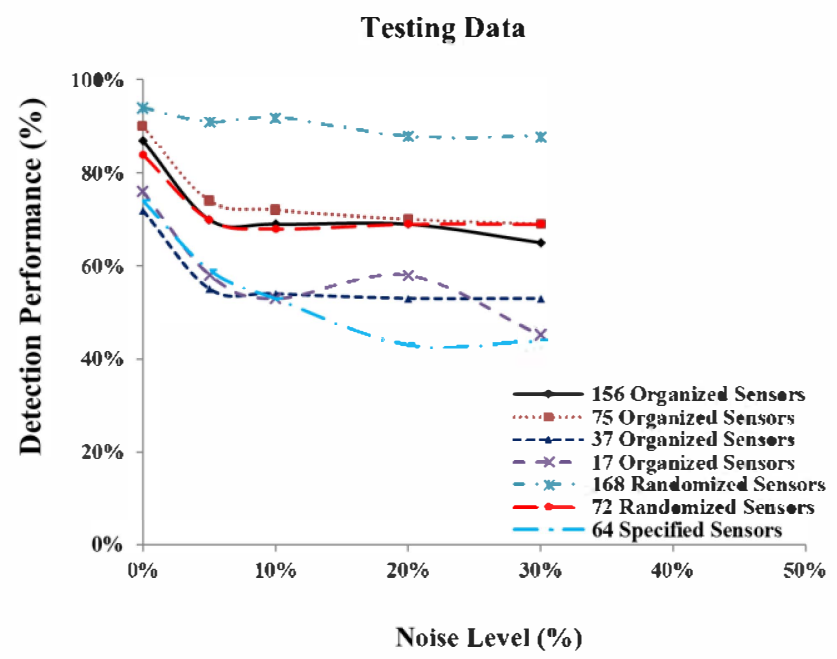

Fig. 5. The damage detection accuracy of the best models versus the noise levels for different number of sensors

\section{CONCLUSIONS}

The present study proposes a new approach for structural damage detection in infrastructure systems based on the simulation of the SWS data. A probabilistic method is combined with FE to analyze the cumulative time distributions at preselected discrete strain levels. Various descriptive features are extracted from the cumulative limited compressed static strain data and then input to the PNN models. Based on the results of the beam analysis, the models developed using coordinates of the sensors, $\mu$ and $\sigma$ do not provide good detection accuracy. Also, the precision of the models expectedly decreases with a decreasing number of sensors. Thus, new predictor features (i.e., $\mu_{D}, \sigma_{D}, \mu_{S}, \sigma_{S}, Z_{\mu 1}, Z_{\sigma 1}, Z_{\mu 2}$, and $Z_{\sigma 2}$ ) are defined that contain the information supplied by all the sensors in each specific sensor configuration. The results indicate that the performance of the models is significantly improved by introducing the sensor group effect, even for a relatively low number of sensors per observation area. Consequently, it is found that $\mu_{D}, \sigma_{D}, \mu_{S}, \sigma_{S}, Z_{\mu 1}, Z_{\sigma 1}, Z_{\mu 2}$, and $Z_{\sigma 2}$ contain useful information for the detection of the damage state in the investigated system. The performance of the method for the gusset plate case is good, especially for higher number of sensors. In order to introduce uncertainty and simulate the performance of real sensors, Gaussian noise is applied to the data. Based on the noise pollution results, the performance of the models remains satisfactory even as the noise level is increased to $20 \%$. 


\section{ACKNOWLEDGMENT}

The presented work is supported by a research grant from the Federal Highway Administration (FHWA) (DTFH61-13-H-00009). The authors are thankful to Dr. Nicholas Brake for his constructive comments.

\section{REFERENCES}

[1]. Sirohi J., I. Chopra, Fundamental Understanding of Piezoelectric Strain Sensors', J. Intell. Mater. Syst. Struct., 11(2001) 246- 257.

[2]. Elvin N., A. Elvin, M. Spector, A self-powered mechanical strain energy sensor. Smart Mater. Struct. 10 (2001) 293-299.

[3]. Lajnef N., K. Chatti, S. Chakrabartty, M. Rhimi, P. Sarkar, Smart
Pavement Monitoring System". Report: FHWA-HRT-12-072, Federal Highway Administration (FHWA), Washington, DC (2013).

[4]. Lajnef N., S. Chakrabartty, N. Elvin, A Piezo-powered Floatinggate Sensor Array for Long-term Fatigue Monitoring in Biomechanical Implants, IEEE Trans. Biomed. Circuits Syst. 2(3) (2008) 164-172.

[5]. Specht D, Probabilistic Neural Networks. Neural Networks 3(1990) 109-118.

[6]. NTSB, Structural and local failure study of gusset plate in minneapolis bridge collapse. Report - NTSBC070010, National Transportation Safety Board (NTSB), Washington, DC (2008). 